\title{
Isolation and Molecular Methods for the Identification of Fusarium solani from Solid Waste
}

\author{
Chetan $\mathrm{DM}^{1^{*}}$, Nataraja $\mathrm{S}^{2}$, Krishnappa $\mathrm{M}^{3}$, Bharath $\mathrm{BR}^{4}$, Ravikumar Patil $\mathrm{HS}^{1}$ and \\ Raghavendra $\mathrm{HL}^{5}$ \\ ${ }^{1}$ Department of Biotechnology NMAM Institute of Technology- Nitte, Karnataka, India \\ ${ }^{2}$ Department of Botany Sahyadri Science College, Kuvempu University-Shimoga, Karnataka, India \\ ${ }^{3}$ Department of studies and research in Applied Botany, Kuvempu University, Shankaraghatta, \\ Karnataka, India \\ ${ }^{4}$ Department of Biotechnology and Bioinformatics, Jnanasahyadri, Kuvempu University, Shankaraghatta, \\ Karnataka, India \\ ${ }^{5}$ College of Health and Medical Sciences, Wollega University, Post Box No: 395, Nekemte, Ethiopia
}

\begin{abstract}
A major goal in microbial ecology is to link specific microbial populations to environmental processes. Solid waste degradation is an imperative aspect of environmental processes mediated by microorganisms individually or symbiotically. In the current study an attempt has been made to isolate and identify a microorganism which can degrade the hazardous xenobiotic compound propaxur commonly known as baygon from solid waste by both in vitro and in silico approaches. 28s rDNA gene has indicated the organism as Fusarium solani. Further studies were extended through bioinformatics approaches like BLAST (Basic Local Alignment Search Tool) and MSA (Multiple Sequence Analysis) to determine the relative phylogeny of this organism. This approach has shed light on evolutionary relationship among the organisms taken in the study. By this approach we have identified the Fusarium solani.
\end{abstract}

Article Information Article History:

Received : 08-01-2013

Revised : 24-03-2013

Accepted : 25-03-2013

Keywords:

28s rDNA

BLAST

MSA

Propoxur

*Corresponding Author:

Chetan DM

E-mail:

chetanmurthy@gmail.com

\section{INTRODUCTION}

"Solid waste" means any garbage, refuse, sludge from a waste treatment plant, water supply treatment plant, or air pollution control facility and other discarded material, including solid, liquid, semisolid, or contained gaseous material resulting from industrial, commercial, mining, and agricultural operations, and from community activities. Total quantity of waste generated in the country (based on weighment exercise by local bodies) is not reported. However, in the year 2000 Ministry of Urban Development in its manual on solid waste management has estimated waste generation of 100,000 MT annually. Central Pollution Control Board (CPCB) with the assistance of National
Environmental Engineering Research Institute (NEERI) has conducted survey of solid waste management in 59 cities (35 metro cities and 24 state capitals 2004-05). Quantities and waste generation rates in 59 cities are as under: In India, the amount of waste per capita generated is estimated to increase at a rate of $1-1.33 \%$ annually (Shekdar, 1999).

Organic waste is a major component of Municipal Solid Waste (MSW), MSW compost contains a significant amount of humic substances. Organic waste is produced wherever and whenever there is human habitation. The main forms of organic waste are household food 
Chetan et al.,

waste, agricultural waste, human and animal waste. In industrialized countries the amount of organic waste produced is increasing dramatically every year. Although many gardening enthusiasts compost some of their kitchen and garden waste, much of the household waste goes into landfill sites and is often the most hazardous waste. The organic waste component of landfill is broken down by micro-organisms to form a liquid 'leachate' which contains bacteria, rotting matter and maybe chemical contaminants from the landfill.

Micro organisms that dwell in solid wastes are grouped under Solid Waste Microflora (SWM). The most common organisms that are generally found in solid waste are bacteria and fungi. These microorganisms use the components of the waste as the substrate for their growth. They grow and multiply on these wastes by utilizing the various components that make up the solid waste. Further a wide variety of pathogenic microorganisms have been reported to be present in these organic wastes (Amalraj et al., 2006).

\section{MATERIALS AND METHODS}

\section{Study Area}

Udupi (Kannada) is a temple town located in Udupi District near Mangalore, Karnataka state, India. As of the 2001 India census Udupi had a population of $1,13,039$. The weather is fairly similar throughout the year, due to the nearby Arabian Sea. Temperature ranges from 30 to $35^{\circ} \mathrm{C}$ in day time and falls by 10 degrees during night and humidity is normally high round the clock with rainy season spanning between April to September.

\section{Collection of Waste Sample}

The Domestic waste about $400 \mathrm{gms}$ was collected in a clean plastic and air tight container of $500 \mathrm{gm}$ capacity. The waste was collected from different residential areas once in a week weekly. Many specific kinds of microorganisms can be allowed to grow from organic wastes by providing the specific optimum environmental conditions which may likely enhance the growth of desired microbes over undesired micro-organisms. Characteristics of the organisms which give them special advantages over other organisms are exploited in the formulation of culture media and the choice of incubation conditions. The collected materials are plated on plastic petri plate as (Standard Blotter Method) SBM and incubated at $28 \pm 2{ }^{\circ} \mathrm{C}$. Observations are done every day under Stereobinocular microscope. The organisms were identified by using Barnett manual (Subramanian, 1983).
Sci. technol. arts Res. J., Jan-Mar 2013, 2(1): $42-49$

\section{Isolation of Fungi}

A fungus was isolated from waste sample using pour plate method. This method is useful for quantifying micro organisms that grow on solid Potato Dextrose Agar (PDA) medium.

\section{Maintenance of Pure Culture}

Isolated fungus was sub cultured on plates and agar slants at regular interval of time to maintain viability and was successfully stored in refrigerators at $4{ }^{\circ} \mathrm{C}$ for $3-4$ months until further studies.

\section{Molecular Methods}

The $\sim 5 \mathrm{~kb}$ rDNA fragment was amplified from isolated genomic DNA using high -fidelity PCR polymerase. The PCR product was sequenced bi-directionally using the forward and reverse primers. The sequence data was aligned and analyzed to identify the whether yeast or fusarium solani and members of its family and distant organisms.

Phylogentic tree builder uses sequences aligned with System Software aligner. A distance matrix is generated using the Jukes-Cantor corrected distance model. When generating the distance matrix alignment model positions are used ignoring the alignment inserts and the minimum comparable position is 200 . The tree is created using Weighbor with alphabet size 4 and length size 1000 .

Weighbor Tree: Weighbor is a weighted version of Neighbor Joining, that gives significantly less weight to the longer distances in the distance matrix. The weights are based on variances and covariances expected in a simple Jukes-Cantor model.

The phylogenetic tree was validated using Bootstrap method, this statistical method used for estimating the sampling distribution by resampling with replacement from the original sample. In making phylogenetic trees, the approach is to create a pseudo alignment by taking random positions of the original alignment. Some columns of the alignment could be selected more than once or not selected at all. The pseudo-alignment will be as long as the original alignment and will be used to create a distance matrix and a tree. The process is repeated 100 times and a majority of consensus tree is displayed showing the number (or percentage) of times a particular group was on each side of a branch without concerning the sub grouping. 
Chetan et al.,

\section{In silico Methods}

To substantiate the in vitro methods for the identification of unknown organism in silico sequence analysis was carried out. For sequence analysis the orthologs sequences for unknown query sequence were retrieved by performing Basic Local Alignment Search Tool (BLAST) (http://blast.ncbi.nlm.nih.gov/Blast.cgi) against nonredundant databases. BLAST finds the regions of local similarity between sequences. The program compares nucleotide or protein sequences to sequence databases and calculates the statistical significance of matches. BLAST can be used to infer functional and evolutionary relationships between sequences as well as help identify members of gene families.
Sci. technol. arts Res. J., Jan-Mar 2013, 2(1): 42-49

The most similar ortholog sequences were retrieved in FASTA format as an input for MSA. ClustalW2 is a general purpose multiple sequence alignment program for DNA or proteins. It produces biologically meaningful multiple sequence alignments of divergent sequences. It calculates the best match for the selected sequences, and lines them up so that the identities, similarities and differences can be seen. Evolutionary relationships can be seen via viewing Cladograms or Phylograms, ClustalW2 was employed for MSA and phylogenetic analysis by setting the gap opening and gap extension parameters as 1 and 0.5 respectively. The tool Tree view V1.6.6 was used to visualise the tree given by ClustalW2 in .ph format.

\section{RESULTS}

\section{Seq 1. The unknown sequence in FASTA format: (2956 bp)}

\section{>Unknown}

AGGGAGAAAGAACCAACAGGGATTGCCCTAGTAACGGCGAGTGAAGCGGCAACAGCTCAAATTTGAAATCTGGCTCTCGGGC CCGAGTTGTAATTTGTAGAGGATACTTTTGATGCGGTGCCTTCCGAGTTCCCTGGAACGGGACGCCATAGAGGGTGAGAGCCC CGTCTGGTTGGATGCCAAATCTCTGTAAAGTTCCTTCAACGAGTCGAGTAGTTTGGGAATGCTGCTCTAAATGGGAGGTATATG TCTTCTAAAGCTAAATACCGGCCAGAGACCGATAGCGCACAAGTAGAGTGATCGAAAGATGAAAAGCACTTTGAAAAGAGAGTT AAAAAGTACGTGAAATTGTTGAAAGGGAAGCGTTTATGACCAGACTTGGGCTTGGTTAATCATCTGGGGTTCTCCCCAGTGCAC TTTTCCAGTCCAGGCCAGCATCAGTTTTCCCCGGGGGATAAAGGCGGCGGGAATGTGGCTCTCTTCGGGGAGTGTTATAGCC CACCGTGTAATACCCTGGGGGGGACTGAGGTTCGCGCATCTGCAAGGATGCTGGCGTAATGGTCATCAACGACCCGTCTTGA AACACGGACCAAGGAGTCGTCTTCGTATGCGAGTGTTCGGGTGTCAAACCCCTACGCGTAATGAAAGTGAACGCAGGTGAGA GCTTCGGCGCATCATCGACCGATCCTGATGTTCTCGGATGGATTTGAGTAAGAGCATACGGGGCCGGACCCGAAAGAAGGTG AACTATGCCTGTATAGGGTGAAGCCAGAGGAAACTCTGGTGGAGGCTCGCAGCGGTTCTGACGTGCAAATCGATCGTCAAATA TGGGCATGGGGGCGAAAGACTAATCGAACCTTCTAGTAGCTGGTTTCCGCCGAAGTTTCCCTCAGGATAGCAGTGTTGAACTC AGTTTTATGAGGTAAAGCGAATGATTAGGGACTCGGGGGCGCTATTTAGCCTTCATCCATTCTCAAACTTTAAATATGTAAGAAG CTCTTGTTGCTTAATTGAACGTGAGCATTCGAATGTATCAACACTAGTGGGCCATTTTTGGTAAGCAGAACTGGCGATGCGGGA TGAACCGAACGCGAGGTTAAGGTGCCAGAGTAGACGCTCATCAGACACCACAAAAGGTGTTAGTACATCTTGACAGCAGGACG GTGGCCATGGAAGTCGGAATCCGCTAAGGACTGTGTAACAACTCACCTGCCGAATGTACTAGCCCTGAAAATGGATGGCGCTC AAGCGTCTCACCCATACCTCGCCCTCAGGGTAGAAACGATGCCCTGAGGAGTAGGCGGACGTGGAGGTCAGTGACGAAGCCT AGGGCGGAGCCCCGGTTGAACGGCCTCTAGTGCAGATCTTGGTGGTAGTAGCAAATACTTCAATGAGAACTTGAAGGACCGAA GTGGGGAAAGGTTCCATGTGAACAGCGGTTGGACATGGGTTAGTCGATCCTAAGCCATAGGGAAGTTCCGTTTCAAAGGCGCA CTATGCGCCGTCTGGCGAAAGGGGAGCCGGTCAATATTCCGGCACCTGGATGTGGGTTTTGCGCGGCAACGCAACTGAACGC GGAGACGACGGCGGGGGCCCCGGGCAGAGTTCTCTTTTCTTCTTAACAGTCTCTCACCCTGAAATCGGTTTGTCCGGAGCTAG GGTTTAATGGCTGGAAGAGCCCAGCACCTCTGCTGGGTCCGGTGCGCTCTCGACGTCCCTTGAAAATCCGCGGGAAGAAATA ATTCTCACGCCAGGTCGTACTCATAACCGCAGCAGGTCTCCAAGGTGAACAGCCTCTGGTTGATAGAACAATGTAGATAAGGG AAGTCGGCAAAATAGATCCGTAACTTCGGGATAAGGATTGGCTCTAAGGGTTGGGCACGCAGGGCCTTGGGCGGACGCCATG GGGGCAGGCTGCTTCTAGCCGGGCAACCGGCCGGCGGCGGCCAGCACCCGTGCGCTGATGCCCTTGGCAGGCTTCGGCCG TCCGGCGTGCGGTTAACAACCAACTTAGAACTGGTACGGACAAGGGGAATCTGACTGTCTAATTAAAACATAGCATTGCGATG GCCAGAAAGTGGTGTTGACGCAATGTGATTTCTGCCCAGTGCTCTGAATGTCAAAGTGAAGTAATTCAACCAAGCGCGGGTAA ACGGCGGGAGTAACTATGACTCTCTTAAGGTAGCCAAATGCCTCGTCATCTAATTAGTGACGCGCATGAATGGATTAACGAGAT TCCCACTGTCCCTATCTACTATCTAGCGAAACCACAGCCAAGGGAACGGGCTTGGCAGAATCAGCGGGGAAAGAAGACCCTGT TGAGCTTGACTCTAGTTTGACATTGTGAAAAGACATAGGAGGTGTAGAATAGGTGGGAGCTTCGGCGCGGTGAAATACCACTA CTCCTATTGTTTTTTTACTTATTCAATGAAGCGGGGCTGGATTTACGTCCAACTTCTGGTTTTAAGGTCGTTCGCGGGCCGAGC CGGGTAGAAGACATTGTCAGGTGGGGAAGTTTGGCTGGGGCGGCACATCTGTTAAACCATAAAGCAGGTGTCCTAAGGGGGG CTCATGGAGAACAGAAATCTCCAGTAGAACAAAAGGGTAAAAGTCCCCTTGATTTTTGATTTTCAGTGTGAATACAAACCATGAAA GTGTGGCCTATCGATCCTTTAGTCCCTAGACATTTGAGGCTAGAGGTGCCAGAAAAGTTACCACAGGGATAACTGGCTTGTGG CGGCCAAGCTTCATAGCGACGTCGCTTTTTGATCCTTCGATGTCGGCTATTCCTATCATACCGAAGCAGAATTCGGTAAGCGTT GGATTGTTCACCCACTAATAGGGAACGTGAGCTGGGTTTAGACCGTCGTGAGACAGGTTAGTTTTACCGTACTGATGACCTCA CCGCAATGGTAATTGAGCTTAGTTAAAAGGGCAATCGCTCTATTCTAAGAAT 


\section{Isolation and Identification}

Microscopical observations of collected waste material, the organisms were identified according to the Barnett manual. The observation revealed that the growth of fungus on waste was comparatively more than other organisms. The isolation of the waste decomposing fungus was quantified from the solid medium. The isolated fungus was sub-cultured on PDA plates and slants.

It is difficult to identify an organism only by morphological studies, thus genomic DNA was isolated from the organism in pure culture using fungal Genomic DNA Isolation Kit (RKT13) and was subjected for electrophoresis with reference to $1 \mathrm{~kb}$ DNA Ladder (Chromous Cat. No. LAD03)
(Figure 1A). The isolated DNA was used as a template for the PCR amplification of $\sim 5 \mathrm{~kb}$ rDNA fragment using high -fidelity PCR polymerase. The reaction mixture used for the $P C R$ is shown in Table 1. The PCR product was sequenced bidirectionally using the forward and reverse primers as per the profile shown in Table 2. The polymerised sample was subjected for electrophoresis by taking 500bp DNA Ladder (Chromous Cat. No. LAD02) as reference (Figure 1B). The reference ladders for DNA isolation $1 \mathrm{~kb}$ DNA Ladder and PCR amplification 500bp DNA Ladder are shown in Figure 1C and Figure 1D respectively. The sequence data was aligned and analyzed to identify the Yeast and its closest neighbours.

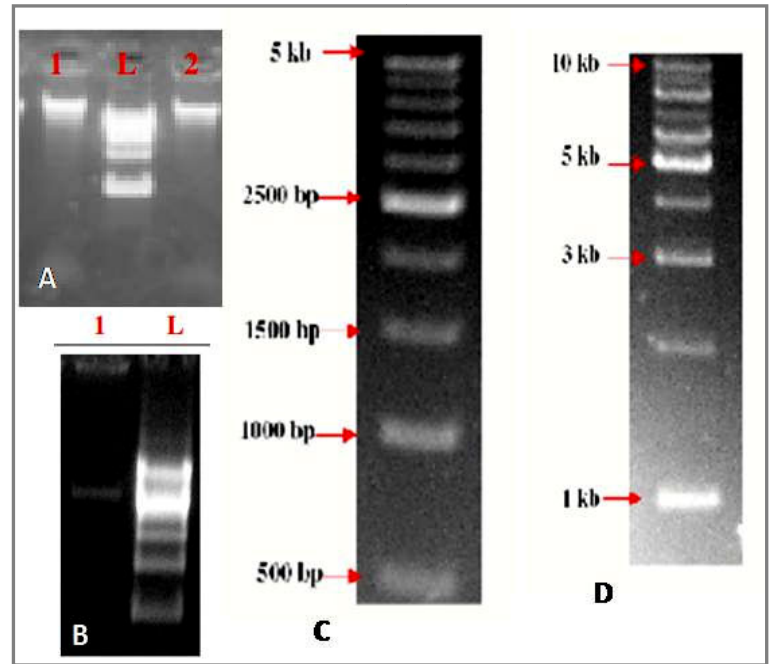

\section{Lane description}

A: L: 1kb DNA Ladder

(Chromous Cat. No. LAD03)

1. Sample

2. Sample

B: L: 500bp DNA Ladder (Chromous Cat. No. LAD02) 1. Sample

C: 500bp DNA Ladder (LAD0

D: 1kb DNA Ladder (LAD03)

Figure 1: Gel images A: Extraction of Genomic DNA from fungal sample using the Fungal Genomic DNA Isolation Kit (RKT13). B: PCR amplification of rDNA fragment from fungal sample. The size of PCR amplified product is $\sim 500$ bp. C: 500 bp ladder contains 10 DNA fragments of size 500 , 1000, 1500, 2000, 2500, 3000, 3500, 4000, 4500 and 5000 bp D: 1 kb ladder contains 10 DNA fragments of size $1,2,3,4,5,6,7,8,9$ and $10 \mathrm{~kb}$.

Table 1: Sequences selected for MSA: Information of gene sequences taken for MSA, information contains the organism name from which the sequenses were retrieved and the length of the sequences.

\begin{tabular}{cll}
\hline Sequence format & \multicolumn{1}{c}{ Pearson } \\
\hline Sequence number & Organism & Length \\
\hline Sequence 1 & Hypocrea-jecorina & $5556 \mathrm{bp}$ \\
Sequence 2 & Metrahizium-anisopliae & $8118 \mathrm{bp}$ \\
Sequence 3 & Tetracladium-marchalianum & $5218 \mathrm{bp}$ \\
Sequence 4 & Tetracladium-breve & $5236 \mathrm{bp}$ \\
Sequence 5 & Tetracladium-palmatum & $5219 \mathrm{bp}$ \\
Sequence 6 & Neurospora-crassa & 8847 bp \\
Sequence 7 & Verticillium-dahliae & $7216 \mathrm{bp}$ \\
Sequence 8 & Magnaporthe-grisea & 8412 bp \\
Sequence 9 & Fusarium-solani & $3830 \mathrm{bp}$ \\
Sequence 10 & Unknown & $1349 \mathrm{bp}$ \\
\hline
\end{tabular}


Table 2: Distance matrix with Sample sequence taken as reference sequence.

\begin{tabular}{cll}
\hline NCBI Accession No. & Organism Name & Score \\
\hline FJ345352 & Fusarium solani & 97 \\
AF510497 & Hypocrea jecorina & 95 \\
AF218207 & Metarhizium anisopliae & 95 \\
FJ360521 & Neurospora crassa & 93 \\
DQ493955 & Magnaporthe grisea & 92 \\
AF104926 & Verticillium dahliae & 90 \\
EU883418 & Tetracladium breve & 89 \\
EU883423 & Tetracladium marchalianum & 89 \\
EU883431 & Tetracladium breve & 89 \\
EU883424 & Tetracladium palmatum & 89
\end{tabular}

Score: Sequence Match Score obtained based on nucleotide alignment.

\section{Identification by In silico Methods}

In order to verify and to substantiate the identification of an unknown solid waste degrading organism as Fusarium solani the most powerful insilico tool i.e phylgogenetic analysis has been employed. Here the sequence obtained from the sequencing was taken as a query sequence. The orthologs sequences for query sequence were retrieved by performing BLAST against non-redundant databases. Phylogram was obtained by performing MSA of all the ten sequences with query sequence shown in Table 3 using the tool ClustalW. The pylogram obtained by performing MAS is shown in Figure 2 . Distance between all the orthologs with sample unknown sequence taken as reference sequence is given in Table 4. Later the alignment gave us information about the conservation level of unknown gene through genes from different organisms. The unknown sequence has shown very good conservation with all the 28s rDNA sequences from different organisms taken for the study as shown in Figure 3. The phylogenetic tree was validated by bootstraping; this analysis supported the identification of an unknown organism as Fusarium solani.

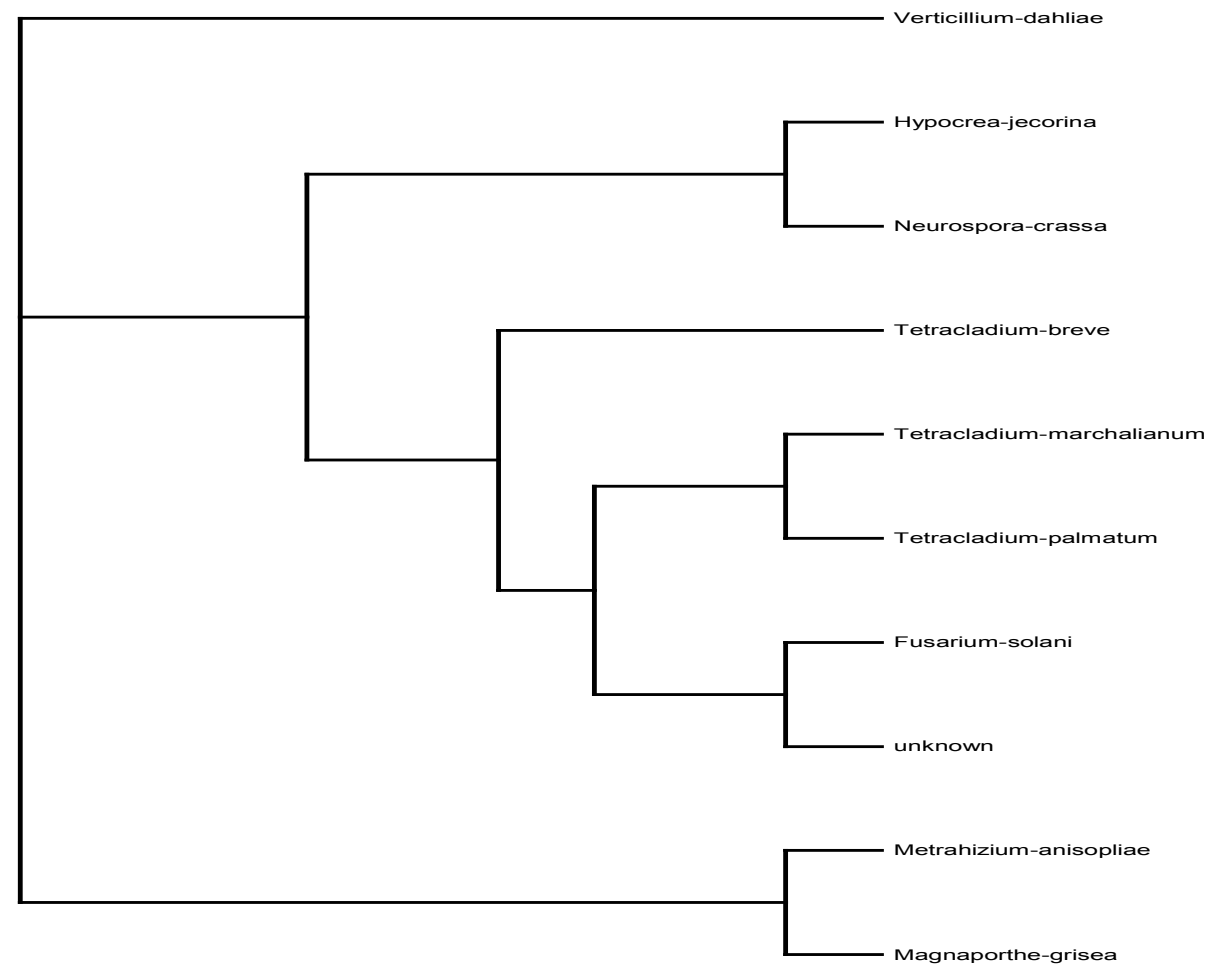

Figure 2: Phylogram obtained from ClustalW2 after performing MSA. Unknown and Fusarium solani were observed in a single clade. 
Chetan et al.

Hypocrea-jecorina Metrahizium-anisopliae Tetracladium-marchalianu Tetracladium-breve Netracladium-palma Verticillium-dahliae Magnaporthe-grisea Fusarium-solani unknown

Clustal Consensus

Hypocrea-jecorina Metrahizium-anisopliae Tetracladium-marchalianum Tetracladium-breve Tetracladium-palmatur Neurospora-crassa Verticillium-dahliae Magnaporthe-grisea Fusarium-solan. unknow

Clustal Consensus

Hypocrea-jecorina Metrahizium-anisopliae Tetracladium-breve Tetracladium-palmat Neurospora-crassa Magnaporthe-grisea Fusarium solani unknown Consensus Hypocrea-jecorina
Metrahizium-anisopliae Tetracladium-marchalian Tetracladium-breve Tetracladium-palmatu Neurospora-crassa Magnaporthe-gri unknow Clustal Consensus

Hypocrea-jecorina Metrahizium-anisopliae Tetracladium-marchalia Tetracladium-breve Tetracladium-palmat Neurospora-crassa Verticillium-dahlia Magnaporthe-gris unknown

clustal Consensus Hypocrea-jecorina
Metrahizium-anisopliae Tetracladium-marchali Tetracladium-breve Neurospora-crassa Verticillium-dahlia Magnaporthe-grisea
Fusarium-solani Fusarium

unknown

Hypocrea-jecorina Metrahizium-anisopliae Tetracladium-breve Tetracladium-palmatu Neurospora-crassa Fusarium-solani unknow clustal Consensus

Hypocrea-jecorina Metrahizium-anisopliae Tetracladium-breve Tetracladium-palmatum Verticillium-dahliae Magnaporthe-grisea Fusarium-solani unknown clustal Consensus

Sci. technol. arts Res. J., Jan-Mar 2013. 2(1): 42-49

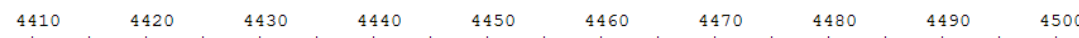
ACCTCGGATCAGGTAGGAATACCCGCTGAACTTAAGCATATCAATAAGCGGAGGAAAGAACCAACAGGGATTGCCCCAGTAACGGCGAGTGAAGCGGC ACCTCGAATCAGGTAGGACTACCCGCTGAACTTAAGCATATCAATAAGCGGAGGAAAAGAAACCAACAGGGATTGCCCCAGTAACGGCGAGTGAAGCGGC ACCTCGGATTAGGTAGGGATACCCGCTGAACTTAAGCATATCAATAAGCGGAGGAAAAGAAACCAACAGGGATTGCCTCAGTAACGGCGAGTGAAGCGGC ACCTCGGATTAGGTAGGGATACCCGCTGAACTTAAGCATATCAATAAGCGGAGGAAAAGAAACCAACAGGGATTGCCTCAGTAACGGCGAGTGAAGCGGC ACCTCGGATTAGGTAGGGATACCCGCTGAACTTAAGCATATCAATAAGCGGAGGAAAAGAAACCAACAGGGATTGCCTCAGTAACGGCGAGTGAAGCGGC ACCTCGGATTAGGTAGGGATACCCGCTGAACTTAAGCATATCAATAAGGGGAGGAAAAGAAACCAACAGGGATTGCCTCAGTAACGGCGAGTGAAGCGGC ACCTCGGATCAGGTAGAATACCCGCTGAACTTAAGCATATCAATAAGCGGAGGAAAAGAAACCAACAGGGATTGCCCTAGTAACGGCGAGTGAAGCGGC ACCTCGGATCAGGTAGGAATACCCGCTGAACTTAAGCATATCAATAAGCGGAGGAAAAGAAACCAACAGGGATTGCCCCAGTAACGGCGAGTGAAGCGGC ACCTCGAATCAGGTAGGAATACCCGCTGAACTTAAGCATATCAATAAGCGGAGGAAAAGAAACCAACAGGGATTGCCCCAGTAACGGCGAGTGAAGCGGC AG-GGAG--AAAGAA-CCAACAGGATTGCCCTAGTAACGGCGAGTGAAGCGGC

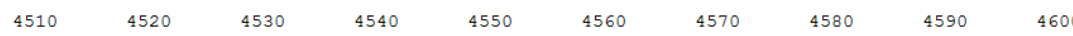
$\ldots \ldots|\ldots| \ldots|\ldots| \ldots|\ldots| \ldots|\ldots| \ldots|\ldots| \ldots|\ldots| \ldots|\ldots| \ldots|\ldots| \ldots|\ldots| \ldots|\ldots| \ldots|\ldots| \ldots|\ldots|$ AACAGCTCAAATTTGAAATCTGGCCCTTTC-GGGTCCGAGTTGTAATTTGTAGAGGATGCTTTTG-G-CAAGGCGCCG-C-CC-GAGTTCCCTGGAACGG AAAAGCTCAAATTTGAAATCTGGCTCTTTTAGGGTCCGAGTTGTAATTTGTAGAAGATG-TTTCG-GGTGTGGCTCCGGT-TT-AAGTTCTTTGGAATAT AAAAGCTCAAATTTGAAATCTGGCTCTTTTAGGGTCCGAGTTGTAATTTGTAGAAGATG -TTTCG - GGTGTGGCTCCGGT - TT -AAGTTCTTTGGAATAT AAAAGCTCAAATTTGAAATCTGGCTCTTTTAGGGTCCGAGTTGTAATTTGTAGAAGATG -TTTCG-GGTGTGGCTCCGGT-TT-AAGTTCTTTGGAATAT | AACAGCTCAAATTTGAAATCTGGCT-T--C--GGCCCGAGTTGTAATTTGTAGAGGAAGCTTTTG-G-TGAGGCACC - - -TCTGAGTCCCCTGGAACGG AACAGCTCAAATTTGAAATCTGGCTCCTTCGGGGTCCGAGTTGTAATTTGTAGAGGATG -CTTCGAGTTATGGTTCC--T-TCCGAGTTCCCTGGAACGG AACAGCTCAAATTTGAAATCTGGCCCCC-C--GGCCCGAGTTGTAATTTGCAGAGGATGCTTTTG-G-TGAGGCACC--TACC-GAGTCCCCTGGAATGG AACAGCTCAAATTTGAAATCTGGCTCT - C-GGGCCCGAGTTGTAATTTGTAGAGGATGCTTTTG-G-TGAGGTGCC--T-TCCGAGTTCCCTGGAACGG AACAGCTCAAATTTGAAATCTGGCTCT-- C-GGGCCCGAGTTGTAATTTGTAGAGGATACTTTTG-A-TGCGGTGCC--T-TCCGAGTTCCCTGGAACGG

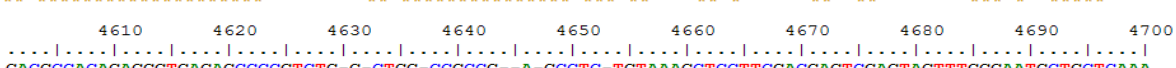
GACGCCA AGAGGGTGAGAGCCCCGTCTG-G-TTGGAT-ACCGA GCCTC-TGTAAAGCTCCTTCGACGAGTCGAGTAGTTTGGGAATGCTGCTCTAA TACATCATAGAGGGTGAGAATCCCGTATGTGACCGG- CAGCCT-TCGCCTA-TGTGAAACTCTTTCGACGAGTCGAGTTGTTTGGGAATGCAGCTCAAA TACATCATAGAGGGTGAGAATCCCGTATGTGACCGG CAGCCT-TCGCCTA-TGTGAAACTCTTTCGACGAGTCGAGTTGTTTGGGAATGCAGCTCAAA GGCGCCATAGAGGGTGAGAGCCCCGTATA-G-TCGGAT-GCCGA-T-CCAA-TGTAAAGCTCCTTCGACGAGTCGAGTAGTTTGGGAATGCTGCTCAAA GACGCCATAGAGGGTGAGAGCCCCGTCTG-G-TAGGA-AACCA--T-GC-TCATGTGAAGCTCCCTCGACGAGTCGAGTAGTTTGGGAATGCTGCTCTAA GGCGCCATAGAGGGTGAGAGCCCCGTATG - G-TAGGAC-GCCGA ACCTC-TGTAAAGCTCCTTCGACGAGTCGAGTAGTTTGGGAATGCTGCTCTAA GACGCCATAGAGGGTGAGAGCCCCGTCTG - G-TTGGAC-ACCGA-T-CCTC-TGTAAAGCTTCTTCGACGAGTCGAGTAGTTTGGGAATGCTGCTCTAA GACGCCATAGAGGGTGAGAGCCCCGTCTG-G-TTGGAT-GCCAAAT-- C-TC-TGTAAAGTTCCTTCAACGAGTCGAGTAGTTTGGGAATGCTGCTCTAA

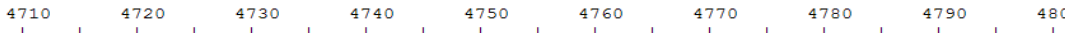

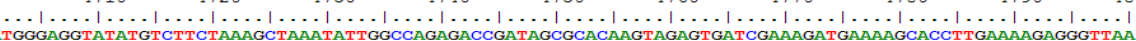
ATGGGAGGTATATGTCTTCTAAAGCTAAATATTGGCCAGAGACCGATAGCGCACAAGTAGAGTGATCGAAAGATGAAAAGCACTTTGAAAAAGAGGGTTAA ATGGGAGGTATATTTCTTCTAAAGCTAAATATTGGCCAGAGACCGATAGCGCACAAGTAGAGTGATCGAAAGATGAAAAGCACTITGGAAAGAGAGTTAA ATGGGAGGTATATTTCTTCTAAAGCTAAATATTGGCCAGAGACCGATAGCGCACAAGTAGAGTGATCGAAAGATGAAAAGCACTTTGGAAAGAGAGTTAA ATGGGAGGTATATTTCTTCTAAAGCTAAATATTGGCCAGAGACCGATAGCGCACAAGTAGAGTGATCGAAAGATGAAAAGCACTTTGGAAAGAGAGTTAA ATGGGAG ATGGGAGGTATA TCCAAGCIAAATACCGGTTAGAGACCGATAGCGCACAAGTAGAGTGATCGAAAGATGAAAAGCACTTTGAAAAGAGAGTCAA ATGGGAGGTAAATTTCTTCTAAAGCTAAATACCGGCCAGAGACCGATAGCGCACAAGTAGAGTGATCGAAAGATGAAAAAGCACTTTGAAAAGAGGGTTAA ATGGGAGGTATATGTCTTCTAAAGCTAAATACCGGCCAGAGACCGATAGCGCACAAGTAGAGTGATCGAAAGATGAAAAGCACTTTGAAAAGAGAGTTAA

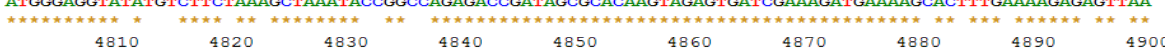

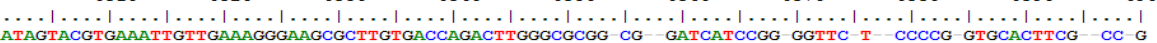
ATAGTACGTGAAATTGTTGAAAGGGAAGACTTATGACCAGACTTGGCCCCG-TG-AATCATCCAGCGGTTC $\sim$ C-CCGTGTGCACTTTG-CC-G ACAGTACGTGAAATTGITGAAAGGGAGCGTTTGCAACCAGACTIGCACGCAG-TT-GATCATCCGG-TGTTC-T-CACCG-GGGCACTCTG-CT-G ACAGTACGTGAAATTGTTGAAAGGGAAGCGTTTGCAACCAGACTTGCACGCAG TT-GATCATCCGG-TGTTC-T-CACCG-GGGCACTCTG-CT-G ACAGTACGTGAAATTGTTGAAAGGAAGCGCTTGCAACCAGACTTGCACGCAG-TT--GATCATCCGG-TGTTC-T--CACCG-GGGCACTCTG--CT-G ATAGCACGTGAAATTGTTGAAAGGAAACGTTTGTGACCAGACTTGCGC-C- - TTCCGATCATCCGG-TGTTC-T--CACCG-GTGCACTCGGGAC--G ACAGCACGIGAAATTGTTAAAAGGGAACGCTCGCTACCAGACGIGGGITCG-TG -GTTCAACCAG-GICCATGAC-CTG-GGGCACTCCG-CC-G AAAGIACGIAAT AAAGTACGTGAAATTGTG AAAGTACGTGAAATTGTTGAAAGGGAGCGTTTATGACCAGACTTGGGCTTGG TT- AATCATCTGG-GGTTC-T- CCCCA-GTGCACTTTT- CC

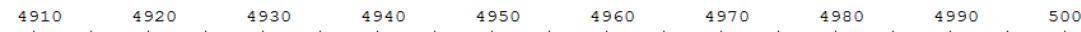
T-GTCCAGGCCAGCATCAG - TTCGTCG C-G-GG GGAAAAGGCTTCGGG AACGTGGCTC-CCCT GGGAGTGTTATAGCCGTTG CATAATA GGGTTCAGGCCAGCATCAG - TTCGCTC-C-G-GG-GGATAAAGGCTTTGGG-AATGTGGCTC-CCTC GGGAGTGTTATAGCCCATTG-CGCAATA C-GTTCAGGCCAGCATCGG-TT-TTG-GTGGTTGGATAAAGGCCTTGGG-AATGTGGCTT-CCTTCG-GGGAGTGTTATAGCCC-TCGG-TGCAATG C-GTTCAGGCCAGCATCGG-TT-TTG-GTGGTTGGATAAAGGCCTTGGG-AATGTGGCTT-CCTTCG-GGGAGTGTTATAGCCC-TCGG-TGCAATG C-GTTCAGGCCAGCATCGG - TT-TTG--GTGGTTGATAAAGGCCTTGGG-AATGTGGCTT-CCTTCG-GGGAGTGT TATAGCCC-TCGG-TGCAATG GCTCAGGCCAGCATCGG - TT-TTGGC-GGGG-GGATAAAGGTC-CGGGGAACGTAGCT--CCTCC--GGGAGTGTTATAGCCC--CGGGCGTAATG GCCCAGGCCAGCATCAGCTTT-CCG-TCG-GG-GG-CAAAGGCGTCGGG-AATGTGGCTCTCCTTCGGGGGAGTGTTATAGCCCGTCG--CGTCATA GTTCAGGCCAGCATCGG-TTT-TCG-CCG-GG-GAACAAAGGCTTCGGG-AACGTGGCTC-CTTTCG-GGGAGTGTTATAGCCCGTTG-CGTAATA GCTCAGGCCAGCATCAG -TTCGCCC-T-G-GG-GGATAAAGGCTTCGGG-AATGTGGCTC-TCTCCG GGGAGTGTTATAGCCCGCTG- CGTAATA GTCCAGGCCAGCATCAG-TTTTCCC-C-G-GG- GGATAAAGGGGCGGG-AATGTGGCTC-TCTTCG-GGGAGTGTTATAGCCCACCG- TGTAATA

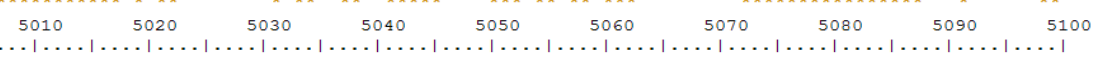
G. C--CCT-G-TGGCGGGCTGAGGTT-CGCGCTTTATGCAAGGATGCTGGCATAATGGTCATCAGTGACCCGTCTTMAAACACGGACCAAGGAGTCGT--CT CAGCCT-A-CCG GGACCGAGGAC-CGCGCTTC-GGCTAGGATGCTGGCGTAATGGTTGTAAACGACCCGTCTTGAAACACGGACCAAGGAGTC-TAACA CAGCCT-A-CCG-GGACCGAGGAC-CGCGCTTC-GGCTAGGATGCTGGCGTAATGGTTGTAAACGACCCGTCTTGAAACACGGACCAAGGAGTC-TAACA C--CCTCG-CCG-GGACCGAGGAC-CGCGCTTC-GGCTAGGATGCTGGCGAATGGT-GIAAGCACCCGTCTTGAAACACGGACCAAGGAGTC-TAACA C--CCT-T-CGGGGGGCTGAGG-TACGCGC-TC-CGCAAGGATGCTGGCGTAATGGTAGCTAGTGACCCGTCTTGAAACACGGACCAAGGAGTCA--ACC C--CCC-GGCGG-GGACCGACGAC-CGCGCTTC-GGCAAGGATGCTGGCGTAATGGTCATCAGCGACCCGTCTTGAAACACGGACCGAGGAGTCA-AGCA C-CCT-G-TGGCGGACTGAGGTT-CGCGCATT-CGCAAGGATGCTGGCGTAATGGTCATCAGTGACCCGTCTTGAAACACGGACCAAGGAGTCGT-CT C--CCT-G-GGGGGACTGAGGTT-CGCGCATC-TGCAAGGATGCTGGCGTAATGGTCATCAACGACCCGTCTTGAAACACGGACCAAGGAGTCGT--CT

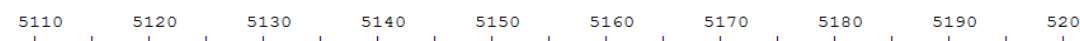

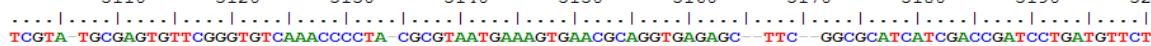
TCGTA-TGCGAGTGTTCGGGTGTCAAACCCTA-CGCGTAATGAAAGTGAACGCAGGTGAGAGC-TTC-GGCGCATCATCGACCGATCCTGATGTTC TCGTA-TGCGAGTGTTCGGGTGTTAAACCCCTA-CGCGTAATGAAAGTGAACGCAGGTGAGAGCCCTCCAGGGCGCATCATCGACCGATCCTGATGTTCT TC-TA-TGCGAGTGTTTGGGTGTTAAACCCATA-CGCGTAATGAAAGTGAACGGAGGTGAGAACCCTTAAGGGTGCATCATCGACCGGTCCTGATGTCT TC-TA-TGCGAGTGTTTGGGTGTTAAACCCATA-CGCGTAATGAAAGTGAACGGAGGTGAGAACCCTTAAGGGTGCATCATCGACCGGTCCTGATGTCTT T--TG-CGCGAGTGTTTGGGTGTAAAACCCGCA-CGCGTAATGAAAGTGAACGTAGGTGAGAGC--TTC--GGCGCATCATCGACCGATCCTGATGTATT TTATG TGCGAGTGCCCGGGCGTAAAACCC-CAGCGCGGAATGAAAGTGAACGTAGGTGAGAGC--TTC- GGCGCATCACCGACCGATCCTGATGTCCT T--TAGTGCGAGTGTTTGGGTGTAAAACCCGCA-CGCGTAATGAAAGTGAACGTAGGTGAGAGC--TTC--GGCGCATCATCGACCGATCCTGATGTTTT

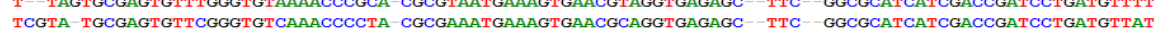
TCGTA-TGCGAGTGTTCGGGTGTCAAACCCCTA-CGCGTAATGAAAGTGAACGCAGGTGAGAGC--TTC- GGCGCATCATCGACCGATCCTGATGTTCT 


Hypocrea-jecorina
Metrahizium-anisopliae
Tetracladium-marchalianum
Tetracladium-breve
Tetracladium-palmatum
Neurospora-crassa
Verticillium-dahliae
Magnaporthe-grisea
Fusarium-solani
unknown
Clustal Consensus

Hypocrea-jecorina Metrahizium-anisopliae Tetracladium-breve Tetracladium-palmatu Neurospora-crassa Magnaporthe-grisea Magnaporthe-gris
Fusarium-solani

Clustal Consensus Hypocrea-jecorina
Metrahizium-anisopliae Tetracladium-marchalianu Tetracladium-breve Tetracladium-palma Verticillium-dahliae Magnaporthe-grisea Fusarium-solani unknown clustal Consensus

Hypocrea-jecorina Metrahizium-anisopliae Tetracladium-marchal Tetracladium-palmatum Neurospora-crassa Verticillium-dahliae Magnaporthe-grisea
Fusarium-solani unknown

clustal Consensus

Hypocrea-jecorina Metrahizium-anisopliae Tetracladium-breve Tetracladium-palmatum Neurospora-crassa verticillium-dahliae Magnaporthe-grisea
Fusarium-solani

unknown

Hypocrea-jecorina Metrahizium-anisopliae Metrahizium-anisopliae
Tetracladium-marchalianum
Tetracladium-breve Tetracladium-palmatum Veurospora-crassa Magnaporthe-grisea Fusarium-solani
unknown

Hypocrea-jecorina Metrahizium-anisopliae Tetracladium-marchalianum Tetracladium-breve Neurospora-crassa Verticillium-dahliae Magnaporthe-grisea
Fusarium-solani unknown

Clustal Consensus

Hypocrea-jecorina Metrahizium-anisopliae Tetracladium-marcha Tetracladium-breve

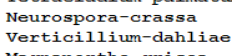
Magnaporthe-grise
Fusarium-solani unknown

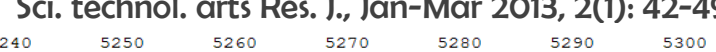

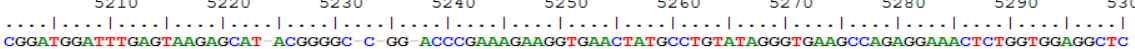
CGGATGGATCTGAGTAAGAGCAT-A GCTGT - GGGACCCGAAAGATG CGGATGGATCTGAGTAAGAGCAT-A-GCTGT - T-GGGACCCGAAAGATGGTGAACTATGCGTGAATAGGGTGAAGCCAGAGGAAACTCTGGTGGAGGCTC CGGATGGATCTGAGTAAGAGCAT - A- GCTGT - T-GGGACCCGAAAGATGGTGAACTATGCGTGAATAGGGTGAAGCCAGAGGAAACTCTGGTGGAGGCTC CGGATGGATTTGAGTAAGAGCGTTA-A- - GCCTTGG-ACCCGAAAGATGGTGAACTATGCTTGGATAGGGTGAAGCCAGAGGAAACTCTGGTGGAGGCTC CGGACGGATTTGAGTGAGAGCAT - ATAGGGT - T-GG-ACCCGAAAGAAGATGAACTATGCGTGTGTAGGGTGAAGCCAGAGGAAACTCTGGTGGAGGCTC CGGA CGGATGGATTTGAGTAAGAGCAT-ACGGGGC-C-GG-ACCCGAAAGAAGGTGAACTATGCCTGTATAGGGTGAAGCCAGAGGAAACTCTGGTGGAGGCTC

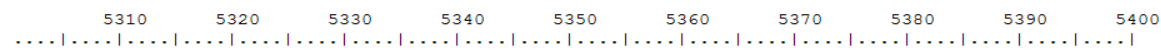
GCAGCGGTTCTGACGTGCAAATCGATCGTCAAATATGGGCATGGGGGCGAAAGACTAATCGAACCTTCTAGTAGCTGGTTTCCGCCGAAGTTTCCCTCAG GCAGCGGTTCTGACGTGCAAATCGATCGTCAAATATGGGCATGGGGGCGAAGACTAATCGAACCTTCTAGTAGCTGGTTTCCGCCGAAGTTTCCCTCAG GCAGCGGTTCTGACGTGCAAATCGATCGTCAAATTTGCGCATAGGGGCGAAAGACTTATCGAACCATCTAGTAGCTGGTTCCTGCCGAAGTTTCCCTCAG GCAGCGGTTCTGACGTGCAAATCGATCGTCAAATTTGCGCATAGGGGCGAAAGACTTATCGAACCATCTAGTAGCTGGTTCCTGCCGAAGTTTCCCTCAG GCAGCGGTTCTGACGTGCAAATCGATCGTCAAATTTGCGCATAGGGGCGAAAGACTTATCGAACCATCTAGTAGCTGGTTCCTGCCGAAGTTTCCCTCAG GCAGCGGTTCTGACGTGCAAATCGATCGTCAAATCTGAGCATGGGGCGAAAGACTAATCGAACCATCTAGTAGCTGGTTACCGCCGAAGTTTCCCTCAG

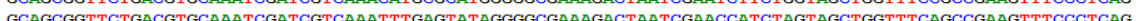

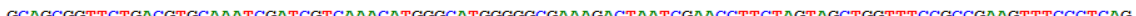
GCAGCGGTTCTGACGTGCDAATCGATCGTCAAATATGGGGATGGGGCGAAABA GCAGCGGTTCTGACGTGCAAATCGATCGTCAAATATGGGCATGGGGGGAAAACTAATCGAACCTTCTAGTAGCTGGTTTCCGCCGAAGTTCCCTCAG

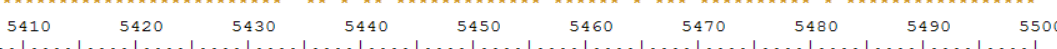
GATAGCAGTGTTGA CTCAGTTTIATGAGGTAAAGCGAATGATTAGGGACC GGGGC GATAGCAGTGTTGATTTCTCAGTTTTATGAGGTAAAGCGAATGATTAGGGAC-CCGGGGC--GG-CTTAT--A- - GCCTTCATCCATTCTCAAACTTTA GATAGCAGTGTTGAA--TTCAGTTTTATGAGGTAAAGCGAATGATTAGAGGCCTTGGGGTT-G-AA-AC-A-ACCTTAACCTATTCTCAAACTTTA GATAGCAGTGTTGAA--TTCAGTTTTATGAGGTAAAGCGAATGATTAGAGGCTTGGGGTT-- G-AA-AC--A--ACCTTAACCTATTCTCAAACTTTA GATAGCAGTGTTGAA--TTCAGTTTTATGAGGTAAAGCGAATGATTAGAGGCCTTGGGGTT- G-AA-AC-A-ACCTTAACCTATTCTCAAACTTTA GATAGCAGTGTTGTTC-TTCAGTTTTATGAGGTAAAGCGAATGATTAGGGAC-TCGGGGGC-G-CT-TTTTA-GCCTTCATCCATTCTCAAACTTTA GATAGCAGTGTTGAT--CCCAGTTTTATGAGGTAAAGCGAATGATTAGGGAC-TCGGGGGCTCGCGCT-ATTTA- GCCTTCATCCATTCTCAAACTTTA GATAGCAGTGTCGTC-TTCAGTTTTATGAGGTAAAGCGAATGATTAGGGA- TCGGGGG-G-AT-TTTTA-GCCTTCATCCATTCTCAAACTTTA GATAGCAGTGTTGAA-CTCAGTTTATGAGGTAAAGCGAATGATTAGGGAC-TCGGGGGC-G-CT-ATTTA-GCCTTCATCCATTCTCAAACTTTA GATAGCAGTGTTGAA--CTCAGTTTATGAGGTAAAGCGATGATTAGGAC-TCGGGGGC-G--CT-ATTTA- GCCTTCATCCATTCTCAAACTTTA

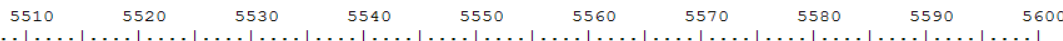

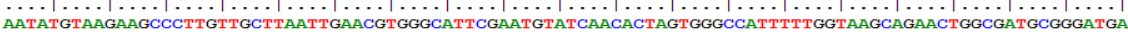
AATATGTAAGAAGCCCTTGTTGCTTAGGTGAACGTGGGCATTCGAATGTATCAACACTAGTGGGCCATTTTTGGTAAGCAGAACTGGCGATGCGGGATGA AATATGTAAGAAGTCCTTGTTACTTAATTGAACGTGGACATTCGAATGTACCAACACTAGTGGGCCATTTTTGGTAAGCAGAACTGGCGATGCGGGATGA AATATGTAAGAAGTCCTTGTTACTTAATTGAACGTGGACATTCGAATGTACCAACACTAGTGGGCCATTTTTGGTAAGCAGAACTGGCGATGCGGGATGA AATATGTAAGAATCCTTGTTACTTAATTGAACGTGGACATTCGAATGTACCAACACTAGTGGGCCATTTTTGGTAAGCAGAACTGGCGATGCGGGATGA AATATGTAAGAAGCCCTTGTTACTTAATTGAACGTGGGCATTCGAATGTACCAACACTAGTGGGCCATTTTTGGTAAGCAGAACTGGGGATGCGGGATGA AATATGTAAGAAGTCCCCGTTACTTATTGAACGCGGACGCTGGAATGAAACAACACTAGTGGGCCATTTTTGGTAAGCAGAACTGGGGATGCGGGATGA AATATGTAAGAAGCCCTTGTTACTTAGTTGAACGTGGGCCTTCGAATGTACCGACACTAGTGGGCCATTTTTGGTAAGCAGAACTGGCGATGCGGGATGA AATATGTAAGAAGCTCTTGTTGCTTAATTGAACGTGAGCATTCGAATGTATCAACACTAGTGGGCCATTTTTGGTAAGCAGAACTGGCGATGCGGGATGA

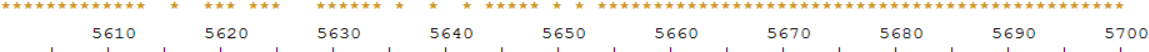

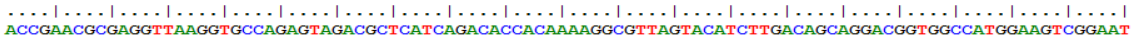
ACCGAACGCGAGGTTAAGGTGCCAGAGTAGACGCTCATCA-ACACCACCCAAAGGTGTTAGTACATCTTGACAGCAGGACGGTGGCCATGGAAGTCGGAAT ACCGAACGTGAAGTTAAGGTGCCGGAATATACGCTCATCAGACACCACAAAAGGTGTTAGTTCATCTAGACAGCAGGACGGTGGCCATGGAAGTCGGAAT ACCGAACGTGAAGTTAAGGTGCCGGAATATACGCTCATCAGACACCACAAAAGGTGTTAGTTCATCTAGACAGCAGGACGGTGGCCATGGAAGTCGGAAT ACCGAACGTGAAGTTAAGGTGCCGGAATATACGCTCATCAGACACCACAAAAGGTGTTAGTTCATCTAGACAGCAGGACGGTGGCCATGGAAGTCGGAAT ACCGAACGCGGGGTTAAGGTGCCGGAGTGGACGCTCATCAGACACCACAAAAGGCGTTAGTACATCTTGACAGCAGGACGGTGGCCATGGAAGTCGGAAT ACCGAACGCGAGGTTAAGGTGCCGGAGTGGACGCTCATCAGACACCACAAAAGGTGTTAGTACATCTT TACAGCAGGACGGT GGCCATGGAAGTCGGAAT ACCGAACGCGGGGTTAAGGTGCCGGAGTGGACGCTCATCAGACACCACAAAAGGCGTTAGTACATCTT TACAGCAGGACGGTGGCCATGGAAGTCGGAAT ACCGAACGCGAGGTTAAGGTGCCAGAGTAGACGCTCATCAGACACCACAAAAGGTGTTAGTACATCTT TACAGCAGGACGGTGGCCATGGAAGTCGGAAT ACCGAACGCGAGGTTAAGTGCCAGAGTAGACGCTCATCAGACACCACAAAAGGTGTTAGTACATCTTGACAGCAGGACGGTGGCATGGAAGTCGGAAT

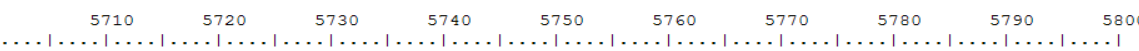

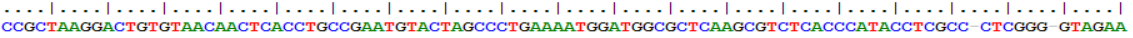
CCGCTAAGGACTGTGTAACAACTCACCTGCCGAATGTACTAGCCCTGAAAATGGATGGCGCTCAAGCGTCTCACCCATACCTCGCC-CTCGGG-GTAGGA CCGCTAAGGAATGTGTAACAACTCACCTGCCGAATGAACTAGCCCTGAAAATGGATGGCGCTTAAGCGTATTACCCATACTTCACCGC-CAGG-GTAGAA CCGCTAAGGAATGTGTAACAACTCACCTGCCGAATGAACTAGCCCTGAAAATGGATGGCGCTTAAGCGTATTACCCATACTTCACCGC-CAGG-GTAGAA CCGCTAAGGAATGTGTAACAACTCACCTGCCGAATGAACTAGCCCTGAAAATGGATGGCGCTTAAGCGTATTACCCATACTTCACCGC-CAGG-GTAGAA

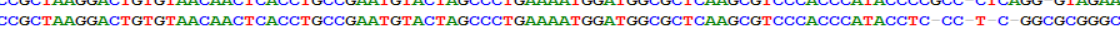
CCGCTAAGGACTGTGTAACAACTCACCTGCCGAATGTACTAGCCCTGAAAATGGATGGCGCTCAAGCGTCCCACCCATACCCCGCC-CCCAGG-GTAGAA CCGCTAAGGACTGTGTAACAACTCACCTGCCGAATGTACTAGCCCTGAAAATGGATGGCGCTCAAGCGTCTCACCCATACCTCGCC-CTCAGG GTAGAA

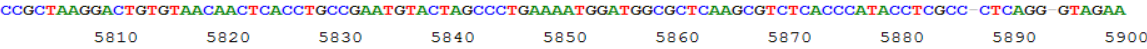
ACGATGC-CCCGAG GAGTAGGCGGACGTGGAGGTC-GTGACGAAGCT AGGGCGTGAGCCCGGGTCGACGGCCTCTAGTGCAGATCTTGGTG GTAG ACGATGC-CCCGAG - GAGTAGGCGACGTGGAGGTC-GTGACGAAGCCT-AGGGCGTGAGCCCGGGTCGAACGGCCTCTAGTGCAGATCTTGGTG GTAG ACGATGC-CCCGAG-GAGTAGGCGGACGTGGGGGTCAGTGACGAAGCCC-AGGGCGTGAGCCCGGGTCGAACGGCCCTAGTGCAGATCTTGGTG-GTAG ACGATGC-CCTG-GCGAGTAGGCAGGCGTGGAGGTCAGTGACGAAGCCTTGGGG- GT GACCCCGGGTAGAACGGCCTCTAGTGCAGATCTTGGTG - GTAG ACGATGC-CCTG-GCGAGTAGGCAGGCGTGGAGGTCAGTGACGAAGCCTTGGG - GTGACCCCGGGTAGAACGGCCTCTAGTGCAGATCTTGGTG-GTAG ACGATGC-CCTGAG-GAGTAGGCGGCCGTGGAGGTCAGTGACGAAGCCT-AGGGCGTGAGCCCGGTTCGAACGGCTCTAGTGCAGATCTTGGTG-GTAG TCGGCGCGTC-GAG -GAGTAGGCGGACGTGGAGGTCGGTGACGAAGCCT-AGGACGTGAGTCCGGGTGGAACGGCCTCTAGTGCAGATCTTGGTG-GTAG ACGATGC-CCTGGG-GAGTAGGCTGACGCGGGGT-AGCGACGAAGGCT-AGGGCGTGAGCCCGGCTAGAGCTGCCCCTGGTGCAGATCTCGGTGAG-AG ACGATGC-CCTGAG-GAGTAGGCGGACGTGGAGTCAGTGACGAAGCCT-AGGGCG -GAGCCCCGGTTGAAC

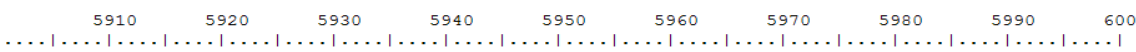
TAGCAAATACTTCAA-TGAGAACTT GAAGGACCGAAGTGGGGAAGGTTCCATGTGACAGCGGTTGGACGTGGGTTAGTCGATCCTAAGCCATAGGGA TAGCAAATACTTCAA-TGAGAACTT - GAAGGACCGAAGTGGGGAAAGGTTCCATGTGAACAGCGGTTGGACGTGGGTTAGTCGATCCTAAGCCATAGGGA TAGCAAATACT - CAAATGAGAACTTTGA-GGACTGAAGTGGGGAAAGGTTCCATGTGAACAGCAGTTGGACATGGGTTAGTCGATCCTAAGCGATAGGGA
TAGCAAATACT-CAAATGAGAACTTTGA-GGACTGAAGTGGGGAAGGTTCCATGTGAACAGCAGTTGGACATGGGTTAGTCGATCCTAAGCGATAGGGA TAGCAAATACT-CAAATGAGAACTTTGA-GGACTGAAGTGGGGAAAGGTTCCATGTGAACAGCAGTTGGACATGGGTTAGTCGATCCTAAGGGATAGGGA TAGCAAATACTTCAA-TGAGAACTT-GAAGGACCGAAGTGGGGAAAGGTTCCATGTGAACAGCGGTTGGACATGGGTTAGTTGAATCCTAAGGCAATAGGGA TAGCAAATACTTCAA-TGAGAACTT-GAAGGACCGAAGTGGGGAAAGGTTCCATGTGAACAGCGGTTGGACGTGGGTCAGTCGATCCTAAGCTATAGGGA TAGCAAATACTTCAA-TGAGAACTT-GAAGGACCGAAGTGGGGAAAGGTTCCATGTGAACAGCAGTTGGACGTGGGTTAGCCGATCCTGAGCCATAGGGA
TAGCAAATACTTCAA-TGAGAACTT-GAAGGACCGAAGTGGGAAAGGTCCATGTGAACAGCGGTTGGACATGGGTTAGTCGATCCTAAGCCATAGGGA TAGCAAATACTTCAA-TGAGAACTT-GAAGGACCGAAGTGGGGAAAGGTTCCATGTGAACAGCGGTTGGACATGGGTTAGTCGATCCTAAGCCATAGGGA

Figure 3: Result of MSA showing the conservation of unkown gene sequence (from $4447^{\text {th }}$ nucleic acid to $5782^{\text {nd }}$ nucleic acid through the sequences of all the organisms taken for study. 


\section{DISCUSSION}

Solid waste was selected for the study to understand diversity of micro flora on solid waste. The most common organisms that are generally found in solid waste are bacteria and fungi. These microorganisms use the components of the waste as the substrate for their growth. So we inoculated the solid waste to the medium suitable for fungal growth. A wide variety of microorganisms have been reported to be present in these organic wastes (Amalraj et al., 2006). Accordingly wide variety of micro flora was observed on the culture medium. An attempt has been made to obtain the pure culture.

By the daily microscopical observations of collected waste material the organisms were identified according to the Barnett manual. It is not easy to identify the microorganisms only by the microscopical observation so the 28s rDNA sequencing method was employed for the identification of the organism on solid waste. The 28s rDNA sequencing was carried out by the isolation and polymerization methods. Genomic DNA was isolated from the organism in pure culture using fungal Genomic DNA Isolation Kit (RKT13) and was subjected for electroporesis with reference to $1 \mathrm{~kb}$ DNA Ladder (Chromous Cat. No. LAD03) (Figure 1A).

The isolated DNA was used as a template for the PCR amplification of $\sim 5 \mathrm{~kb}$ rDNA fragment using high -fidelity PCR polymerase. The PCR product was sequenced bi-directionally using the forward and reverse primers. The polymerised sample was subjected for electrophoresis by taking 500bp DNA Ladder (Chromous Cat.No.LAD02) as reference.

The molecular method of identification was followed by the in silico methods. The orthologs sequences for query sequence were retrieved by performing BLAST against non-redundant databases. Phylogram was obtained by performing MSA of all the ten sequences with query sequence. Both Fusarium solani and unknown sequences were observed on a same clade of phylogram as shown in Figure 2. From the phylogram analysis it was confirmed that the unknown sequence is from Fusarium solani. Later the alignment gave us information about the conservation level of unknown gene through genes from different organisms. The unknown sequence has shown very good conservation from $4447^{\text {th }}$ base to $5872^{\text {nd }}$ base with all the $28 \mathrm{~s}$ rDNA sequences from different organisms taken for the study as shown in Figure 3. The phylogenetic tree was validated by bootstraping; this analysis supported the identification of an unknown organism as Fusarium solani.

\section{CONCLUSION}

The results of the present study reveals that, the differences in Fusarium solani identification by phenotypic and 28 s rDNA sequencing method have shown that an application of a molecular analysis is essential to complement classic biochemical methodology. Our results, together with the review of literature, suggest that $28 \mathrm{~s}$ rDNA sequencing method can be helpful in resolving ambiguous results, for characterizing uncommon strains, and for epidemiological investigations.

The phylogenetic tree was validated by bootstraping; this analysis supported the identification of organism as Fusarium solani through in-silico method. It provides information to facilitate researchers to comprehend the basic views on gene homology and its various applications in the identification of novel strains in microorganism.

\section{REFERENCES}

Amalraj, S. (2006). Introduction to Environamental Science and Technology. Laxmi publication, 61-66.

Shekdar. (1999). Municipal solid waste management. The Indian perspective Journal of Indian Association for Environmental Management, 26, 100-108.

Subramanian CV (1983). Thypomycetes taxonomy and biology. Academic press London, 2. 